\title{
Electroless Gold Plating on Aluminum Patterned Chips for CMOS-Based Sensor Applications
}

\author{
Jung Woo Ko, ${ }^{a}$ Hyo Chol Koo, ${ }^{b}$ Dong Wan Kim, ${ }^{c}$ Sung Min Seo, ${ }^{\mathrm{c}}$ Tae June Kang, ${ }^{\mathrm{d}}$ \\ Yongjoo Kwon, ${ }^{a}$ Jung Lim Yoon, ${ }^{\mathrm{a}}$ Jun Ho Cheon, ${ }^{\mathrm{c}}$ Yong Hyup Kim, ${ }^{\mathrm{d}}$ \\ Jae Jeong Kim, ${ }^{\mathrm{b}, *}$ and Young June Park ${ }^{\mathrm{a}, \mathrm{c}, \mathrm{z}}$
}

${ }^{a}$ NANO Systems Institute-National Core Research Center, ${ }^{b}$ School of Chemical and Biological Engineering, ${ }^{c}$ School of Electrical Engineering, and ${ }^{d}$ School of Mechanical and Aerospace Engineering, Seoul National University, Seoul 151-742, Korea

\begin{abstract}
We presented an approach for the activation of aluminum ( $\mathrm{Al}$ ) alloy using palladium $(\mathrm{Pd})$ and the subsequent gold $(\mathrm{Au})$ electroless plating (ELP) for complementary metal oxide semiconductor (CMOS)-based sensor applications. In this study, CMOS process compatible Al patterned chips were used as substrates for easy incorporation with existing CMOS circuits. To improve the contact resistance that arose from the Schottky barrier between the metal electrodes and the single-walled carbon nanotubes (SWCNTs), electroless deposition of gold that has a higher work function than Al was adopted because the SWCNTs has p-type semiconductor properties. Each step of the Au ELP procedure was studied under various bath temperatures, immersion times, and chemical concentrations. Fine Pd particles were homogeneously distributed on the Al surface by the Pd activation process at room temperature. Au ELP allowed selective deposition of the Au film on the activated Al surface only. The SWCNT networks formed on the Au plated chip by a dip-coating method showed improved contact resistance and resistance variation between the Au electrode and SWCNTs. We also tried SWCNT decoration with the Au particle using the upper Au ELP method, which was expected to be applied in various areas including field-effect transistors and sensor devices. (c) 2009 The Electrochemical Society. [DOI: 10.1149/1.3244208] All rights reserved.
\end{abstract}

Manuscript submitted July 30, 2009; revised manuscript received September 7, 2009. Published November 13, 2009.

Recently, the carbon nanotube (CNT) has been considered a promising candidate for a sensor material due to its high conductivity, small size, high mechanical strength, and stability in a harsh chemical environment. ${ }^{1-7}$ For these sensor materials to be a part of the commodity technology in the future, the integration of the nanomaterial with the complementary metal oxide semiconductor (CMOS) integrated circuit platform is indispensable for lower cost, reduced power consumption, and miniaturization. However, big technical challenges in the integration to CMOS chips are to acquire a reliable method of mounting the CNTs on required positions, and obtaining small contact resistance with aluminum (Al), which is used as a final metal pad for the conventional CMOS process. So, metal plating such as with gold $(\mathrm{Au})$ is needed on top of the $\mathrm{Al} \mathrm{pad}$ which forms the ohmic contact with CNTs.

On the other hand, electroless plating (ELP) of noble metals on $\mathrm{Al}$ has been used in a variety of potential applications including flip-chip bonding of the CMOS chips on the printed circuit board, and protective coatings. ${ }^{8-13}$ Traditionally, Au ELP ${ }^{14}$ on $\mathrm{Al}$ is preceded by the zincate process $(\mathrm{Al} \rightarrow \mathrm{Zn} \rightarrow \mathrm{Ni} \rightarrow \mathrm{Au})$, which activates the $\mathrm{Al}$ surface. ${ }^{15-19}$ The process involves the immersion of the $\mathrm{Al}$ substrate in a strong alkaline zincate solution. This process consists of dissolution of a native $\mathrm{Al}$ oxide and the galvanic displacement reaction between zinc $(\mathrm{Zn})$ ion and $\mathrm{Al}$, and the formed $\mathrm{Zn}$ acts as a catalytic layer of the $\mathrm{Au}$ electroless deposition.

The selective formation of Pd particles directly onto patterned $\mathrm{Al}$ substrates is a potential alternative in zincating processes used as intermediate layers in electrochemical metal coatings. This process has been studied as a replacement of physical vapor deposition of the metal seed layer on a substrate that has a poor step coverage before electroplating some metal interconnects in ultralarge-scale integrated circuit technology. ${ }^{20-22}$

Although the Au ELP technique has long been known, the traditional Au ELP on the Al surface has complicated steps, and to the best of our knowledge, the formed Au surface has not yet been utilized in the sensor applications. In this research, we have studied selective Au ELP on Al patterns with Pd activation to decrease the contact resistance between single-walled carbon nanotubes (SWCNTs) and Al contact pads. The optimization of Pd activation and Au ELP was preceded. Dip coating of SWCNTs, their electrical

\footnotetext{
* Electrochemical Society Active Member

${ }^{\mathrm{z}}$ E-mail: ypark@snu.ac.kr
}

properties, and SWCNT decoration with Au was also tested. We believe our simple Pd activation and Au ELP technique can be used in a wide range of promising applications including CMOS-based field-effect transistors and bio- or gas sensor devices.

\section{Experimental}

All chemicals and solvents used in this experiments were of reagent grade and used without further purification.

The fabrication of the sample chip followed the conventional CMOS fabrication process, which is summarized in Table S1 (see supporting information). ${ }^{30}$ Two kinds of chips were fabricated at the Inter-university Semiconductor Research Center. The substrate was sputtered and patterned to form a 10,000 $\AA \mathrm{Al}$ alloy metal electrode (99.3 wt \% Al, 0.2 wt \% Si, and 0.5 wt $\% \mathrm{Cu}$ ) on a $10,000 \AA$ thick tetraethyl orthosilicate (TEOS) oxide film deposited on a cleaned silicon wafer. Thereafter, two distinctive processes of Au ELP on the $\mathrm{Al}$ surface were used in this paper. Process A followed the patterning of the Al film, chemical Pd activation, and Au ELP (for process B see Fig. S3 in the supporting information). ${ }^{30}$ The detailed process is shown in Fig. 1a.

The Pd activation and Au ELP experiment were investigated for two types of Al patterned chips named M1A, M1B, and M2 (M1 and M2 represent the metal layer number and A and B indicate the pattern type of the sample chips) in this paper (for other M2-type chips, see Fig. S6-7 in the supporting information). ${ }^{30}$ The Pd activation and Au ELP were performed as follows: Before Pd activation, the samples of the $\mathrm{Al}$ patterned chips were cleaned with acetone for 1 min, washed briefly with deionized (DI) water, and dried with a $\mathrm{N}_{2}$ blowing gun. For the chemical Pd activation, Pd activation solutions were prepared by mixing the solutions with the components dissolved. Pd activation solutions were composed of a Pd solution (0.050 $\mathrm{g} \mathrm{PdCl}_{2}, 1.5 \mathrm{~mL} \mathrm{HCl}, 18.5 \mathrm{~mL}$ DI water), a poly(ethylene glycol) (PEG, MW 3400) solution (15 g of PEG in $20 \mathrm{~mL}$ DI water), and a methyl gallate (MG) solution $(0.10 \mathrm{~g}$ of MG in $40 \mathrm{~mL}$ DI water dissolved with sonication). The preparation of the Pd activation solutions followed the next steps: The addition of the PEG solution $(1.0 \mathrm{~mL}), \mathrm{MG}$ solution $(6.0 \mathrm{~mL}), 85 \% \mathrm{H}_{3} \mathrm{PO}_{4}(1.0-4.0$ $\mathrm{mL}$ ), and Pd solution $(0.80 \mathrm{~mL}$ ) to a stirred DI water (adjusted to fit the total volume of the solution to $20 \mathrm{~mL}$ ). The sample was dipped into the solution for an activation time of 1-5 min at room temperature and then it was rinsed in DI water and dried with a $\mathrm{N}_{2}$ blowing gun.

The Au plating solution was prepared by the slow addition of 
(a)

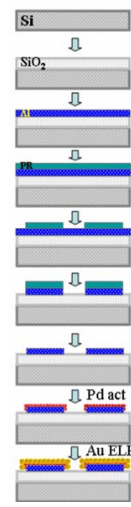

(b)
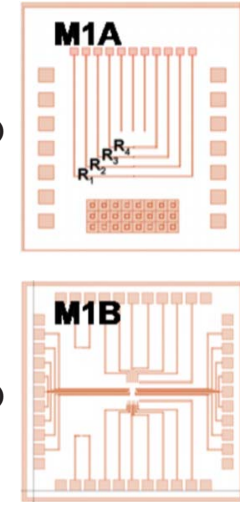

(c)

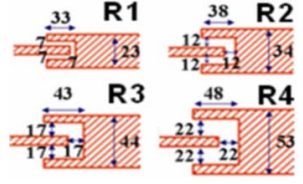

(e)

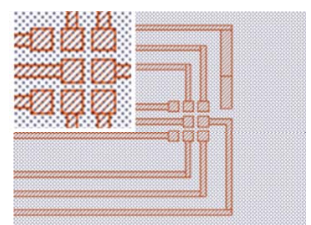

Figure 1. (Color online) (a) The fabrication process of an $\mathrm{Al}$ patterned chip and additional $\mathrm{Pd}$ activation-Au electroless deposition (referred to as process A in this paper). (b) and (c) show the whole part of the chip pattern and the center part of the electrode in the metal layer (M1A), respectively. (d) and (e) show the whole part of the chip pattern and the center part of the electrode in the metal layer (M1B), respectively. The short electrode-electrode distance is $6 \mu \mathrm{m}$.

$0.059 \mathrm{~g} \mathrm{KCN}, 0.91 \mathrm{~g}$ citric acid, and $2.0 \mathrm{~mL}$ hydrazine to a stirred solution of $\mathrm{KAu}(\mathrm{CN})_{2}(0.13 \mathrm{~g})$ in DI water $(50 \mathrm{~mL})$ at room temperature.

The Au ELP was performed with two kinds of Pd-activated Al surfaces by chemical and physical methods. The Pd-activated samples were immersed into the prepared $\mathrm{Au}$ plating solution for 5-180 $\mathrm{min}$, and the temperature varied from 40 to $90^{\circ} \mathrm{C}$. After deposition, the chips were also rinsed in DI water and dried with a $\mathrm{N}_{2}$ blowing gun.

The coating of the SWCNT was done according to the previously reported procedure. ${ }^{23,24}$ The SWCNTs (ASP-100F produced by Iljin Nanotech, Korea) were ultrasonicated in nitric acid at $50^{\circ} \mathrm{C}$ for $30 \mathrm{~min}$ to purify (amorphous carbon and carbonaceous particles were removed) and simultaneously exfoliate from bundles. Then SWCNTs were neutralized with DI water and trapped on the membrane filter (Millipore, $0.2 \mu \mathrm{m}$ pore size and $47 \mathrm{~mm}$ diameter) by a vacuum filtration method. The SWCNTs on the filter were dried in a vacuum oven chamber at $80^{\circ} \mathrm{C}$ for $48 \mathrm{~h}$. The prepared SWCNTs were dispersed in 1,2-dichlorobenzene solution with a concentration of $0.10 \mathrm{mg} / \mathrm{mL}$, and an ultrasonication process is performed for 20 h. Using this solution, the sample chips were coated with the SWCNT net by dipping for $30 \mathrm{~s}$ and withdrawing at the velocity of $3 \mathrm{~mm} / \mathrm{min}$. The amount or density of the SWCNTs was controlled by the SWCNT concentration in the solution, which affects the resistance.

In this research, the surface morphology of the chips was evaluated with an optical microscope (OM) and field-emission-scanning electron microscope (FESEM). The area analysis after Pd activation was performed using an electron probe microanalyzer (EPMA). The measurement of the contact resistance between metal pads and SWCNTs was done using an Agilent 4156C. Currents between the electrodes were measured in the air condition under a voltage sweep of one electrode with the other electrode grounded.

\section{Results and Discussion}

$P d$ activation of annealed chip. - The role of the surface activation is extremely important in electroless deposition because it initiates the deposition reaction and also because it usually functions as a nucleation site for the metal deposition. The conventional process involves oxidation of a substrate for electron generation, which reduces $\mathrm{Pd}$ ions. Furthermore, the negative reduction potential of $\mathrm{Al}$ compared to $\mathrm{Pd}$ allows a spontaneous displacement reaction. However, the existence of the native oxide on the surface is a barrier to all kinds of reaction with pure Al metal, and the oxides cannot be

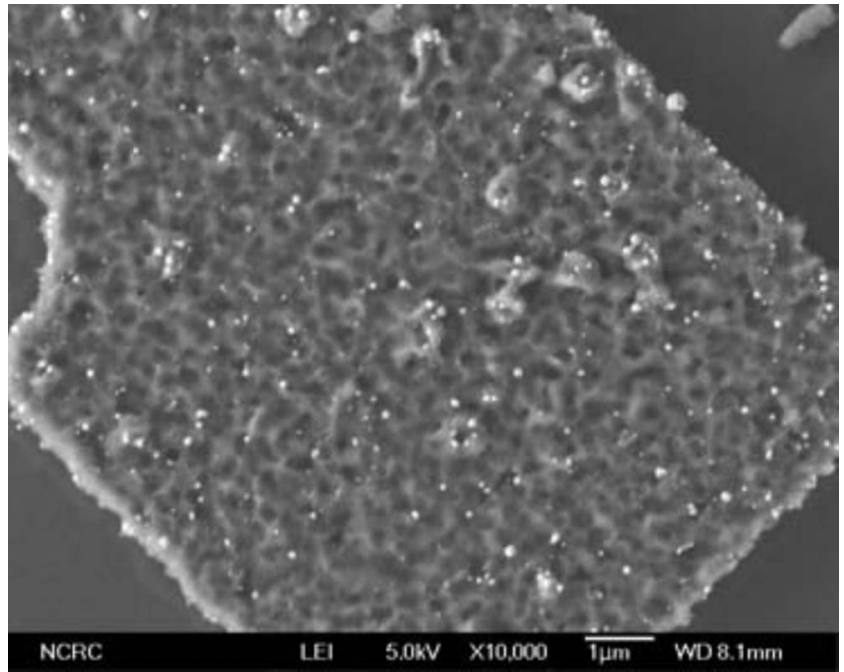

Figure 2. The FESEM image of an Al electrode in M1B chips, which were catalyzed by Pd activation (condition A in Table $\mathrm{S} 2$ ) ${ }^{30}$ It shows the Pd particles scattered randomly on the $\mathrm{Al}$ surface.

removed completely in atmospheric condition. Therefore, removal of the native oxide and reduction of a $\mathrm{Pd}$ ion must be simultaneously accomplished in a bath.

The cleaned Al chips fabricated as shown in Fig. 1 and Table $\mathrm{S} 1^{30}$ were activated with varying phosphoric acid concentrations and immersion time (see Table S2 for supporting information). ${ }^{30}$ The phosphoric acid is expected to remove the native $\mathrm{Al}$ oxide. ${ }^{25}$ After the Pd activation step (conditions A-D), the formation of Pd particles on the Al surface with densities of $10^{9}-10^{10}$ particles $/ \mathrm{cm}^{2}$ was observed, and most of the particles were in the diameter range of $30-50 \mathrm{~nm}$. Abnormally grown larger particles $(120-200 \mathrm{~nm})$ were also observed. The bright fine particles that corresponded to Pd were found in the Al surface, which were confirmed by EPMA (see Fig. $\mathrm{S} 2$ in supporting information). ${ }^{30}$ The morphologies of $\mathrm{Pd}$ particles on the Al electrode were depicted in Fig. 2 and Fig. S1 (see supporting information). ${ }^{30}$ The $\mathrm{Pd}$ activation results depend on the phosphoric acid concentration and activation time. From the view of overall result of the Au ELP associated with the uniform Pd particle density on the Al surface, the Al thickness after Pd activation, and the result of their Au ELP, the optimum condition for Au ELP is listed as the condition A in Table S2. ${ }^{30}$ During the Pd activation, a large amount of Al layers was dissolved, which exceeded over 3000

(a)

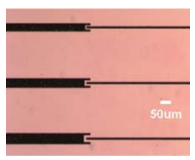

(e)

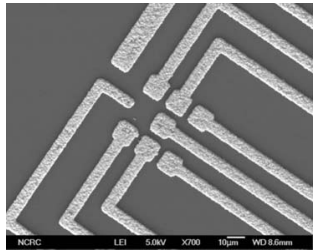

(b)

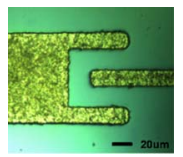

igure 3. (Color online) (a) Middle and (c) lower parts of OM images in M1A's electrode after Au ELP for $2 \mathrm{~h}$ at $50^{\circ} \mathrm{C}$. (b) and (d) show the enlarged electrode of (a) and (c), respectively. (e) Scanning electron microscopy (SEM) and (f) OM images of M1B's electrode after Au ELP for 90 min at $50^{\circ} \mathrm{C}$. (f) shows the enlarged electrode of (e). All Pd-activated samples were prepared immediately before the Au ELP process in condition A. 
(a)

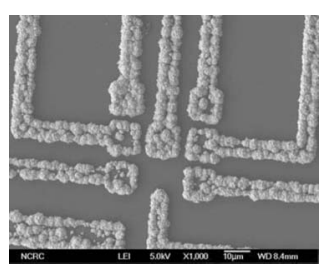

(c)

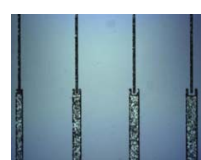

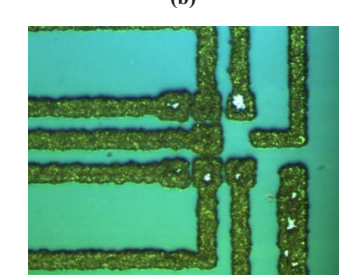

(e)

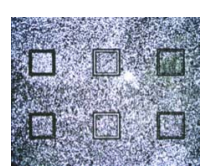

(f)

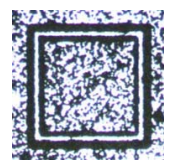

Figure 4. (Color online) The FESEM and OM images of the electrolessly deposited $\mathrm{Au}$ on annealed $\mathrm{Al}$ pattern (M1B) without $\mathrm{Pd}$ activation at $50^{\circ} \mathrm{C}$ for (a) 55 and (b) $100 \mathrm{~min}$, respectively. [(c)-(f)] OM images of the results of Au ELP on Pd-activated M1A (unannealed) sample. The Pd activation condition was A in Table $\mathrm{S}_{2}^{30}$ and Au ELP time was $2 \mathrm{~h}$ at $50^{\circ} \mathrm{C}$. (d) and (f) show the enlarged electrode of (c) and (e), respectively. Black indicates Au.

$\AA$ (condition A). This result implies that the Pd activation process relies on the removal and subsequent dissolution of Al films from the surface of the $\mathrm{Al}$ substrate, with simultaneous reduction of $\mathrm{Pd}$ ions on the $\mathrm{Al}$ surface. Also, very fast dissolution of the film eliminates fore-formed Pd particles from the surface. Pd nucleation was improved by brief treatment of 5:1 (v/v) buffered hydrofluoric acid (BHF, $40 \% \mathrm{NH}_{4} \mathrm{~F}$, and $49 \% \mathrm{HF}$ ) solution before the Pd activation step. Such diluted BHF solution is usually used to strip the thin layer of native Al oxide. ${ }^{24}$ The FESEM analysis indicated that the Pd particles deposited by chemical reaction have a narrow size distribution than the samples deposited by the physical thermal evaporation method (process B in Fig. S4, see supporting information). ${ }^{30}$

Au ELP of chemically Pd-activated M1A and M1B samples.For the annealed $\left(450^{\circ} \mathrm{C}, 30 \mathrm{~m}\right)$ sample, the Pd-activated sample was prepared from the chemical reaction using a Pd activating mixture (condition A), which was prepared immediately before the Au ELP process. Then Au ELP onto Pd-activated samples was done using a Au plating mixture. To know the immersion time and temperature dependence of Au ELP, the plating was carried out at $40-90^{\circ} \mathrm{C}$ for $5-180 \mathrm{~min}$ in a Au plating bath. From the view of the uniformity of the Au particle size, the Au film thickness, and the coverage on the $\mathrm{Al}$ surface, we concluded that optimum bath temperature and plating time for Au ELP are about $50^{\circ} \mathrm{C}$ and ranged from 90 to $120 \mathrm{~min}$, respectively. In this condition, the Au layer is formed with a thickness of approximately $10,000 \AA$, and the electrode gaps are filled up to $2 \mu \mathrm{m}$ by Au ELP. It also passed the Scotch tape adhesion test. The result of the Au ELP in this condition is shown in Fig. 3 (for other M2-type chip, see Fig. S6-S7 in the supporting information).$^{30}$ As expected, the Au coverage or the density on the Al surface increased with increasing plating time at $50^{\circ} \mathrm{C}$. The deposition rates increased with increasing deposition temperature, but the stability of the electrolyte became very poor in the sense that the large clusters of Au were formed in the electrolyte spontaneously.
For a control experiment, Au ELP on the Al pattern without Pd activation was accompanied. The Au ELP results of the M1B sample without $\mathrm{Pd}$ activation at $50^{\circ} \mathrm{C}$ for 55 and 100 minutes indicate that the Au ELP is available even without Pd activation (Fig. 4a and b). One of the suspected reaction pathways is the direct displacement reaction between $\mathrm{Al}$ and $\mathrm{Au}$, and the small composition of $\mathrm{Cu}$ near the $\mathrm{Al}$ surface acts as a catalyst that allows the ELP on the $\mathrm{Al}$ surface without Pd activation. ${ }^{26-28}$ However, the uniformity of the Au film on the $\mathrm{Al}$ surface was poorer than that of the case with Pd activation, and the size of $\mathrm{Au}$ particles constructing the film was quite large, leading to a rough surface morphology. Therefore, it is not appropriate for the application in microelectronic devices in terms of a sophisticated electrode design, which requires a more enhanced substrate pretreatment for the subsequent deposition of gold.

Using the unannealing sample, the annealing effects were examined because it is reported that the native oxide on the as-deposited and annealed $\left(400^{\circ} \mathrm{C}\right.$ for $\left.5 \mathrm{~min}\right) \mathrm{Al}(\mathrm{Cu})$ film was considered negligible compared to the $60-70 \mathrm{~nm}$ thick oxide layer in the asdeposited only $\mathrm{Al}(\mathrm{Cu})$ film. ${ }^{29}$ As shown in Fig. 4c-f Au particles were partially plated on the electrodes after Pd activation (condition A) and $\mathrm{Au}$ ELP process for $2 \mathrm{~h}$ at $50^{\circ} \mathrm{C}$. Also, Au particles were formed along the sidewall rather than on the upper surface of the $\mathrm{Al}$ pads under this condition. It can be speculated that the upper surface of $\mathrm{Al}$ is more contaminated than the sidewall during the patterning process such as photoresist and other various chemicals. The $\mathrm{Al}_{2} \mathrm{O}_{3}$ layer prevents the $\mathrm{Al}$ surface from forming Pd particles in the Pd activation step and the Au film in the subsequent Au ELP step as well. Accordingly, this is not suitable for submicrometer applications.

SWCNT coating and I-V characteristics. - The SWCNTs' surfaces were purified and modified in boiling $\mathrm{HNO}_{3}$. In this step, contaminants were removed and functional groups, such as carboxylic acid, hydroxyl, and carbonyl groups were introduced to the surfaces of the SWCNTs, which induce good dispersion in coating solvent and adhesion on a hydrophilic TEOS layer. The schematic of the SWCNT coating method is shown in Fig. 5a. The SWCNT network is integrated between the electrodes by the dip-coating method, which is randomly distributed on the substrate such as a net (Fig. 5b-d). In Au-plated M1A chip after SWCNT coating, the measured resistances were in the range of $10-40 \mathrm{k} \Omega\left(R_{1}-R_{4}\right)$, depending on the gap length of the electrodes (the short electrode gaps for $R_{1}: 7, R_{2}: 12, R_{3}: 17$, and $\left.R_{4}: 22 \mu \mathrm{m}\right)$. The current-voltage $(I-V)$ characteristics of the device are shown in Fig. S5. ${ }^{30}$ The electrode sweeps $(-1$ to $1 \mathrm{~V})$ are performed in air, and the measured currents are plotted together for comparison. The contact resistance between the SWCNTs and electrodes was improved after Au ELP on the Al electrodes. The average resistance between the electrodes $(25 \mathrm{k} \Omega)$ was reduced to about $2 \times 10^{6}$ times compared to that of the SWCNT-coated Al patterned M1A chip (average of $50 \mathrm{G} \Omega$ ). This suggests that the contact between Au and SWCNTs is ohmic contact compared to the case of $\mathrm{Al}$ and SWCNT, and the Au ELP obviously improved the quality of the contact.

Au particle decoration on SWCNT network.- We assumed that the coated SWCNTs on the oxide layer can be decorated with Au particles. The SWCNT can act as a seed and an electron delivery site, such as the Pd catalyst for SWCNT decoration because (a)

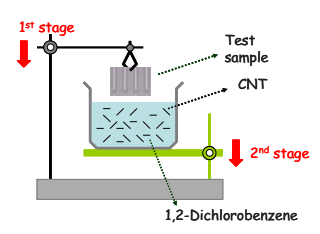

(b)

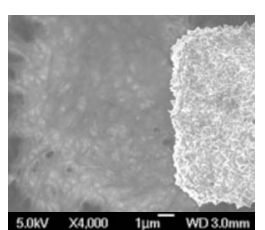

(c)

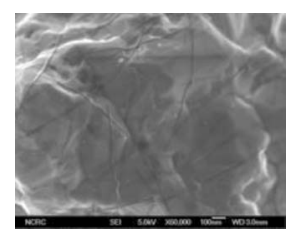

(d)

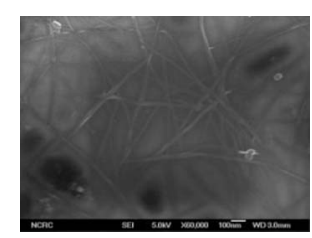

Figure 5. (Color online) (a) Schematic drawing of the dip-coating method of SWCNTs. Dip coating was performed at room temperature by immersing the $\mathrm{Au}$ plated chips vertically in the SWCNT suspension for $30 \mathrm{~s}$ and withdrawing them at a velocity of $3 \mathrm{~mm} / \mathrm{min}$. (b) The SEM image of the (c) Au electrode and the (d) oxide layer on the M1B chip after SWCNT dip coating. 
(a)

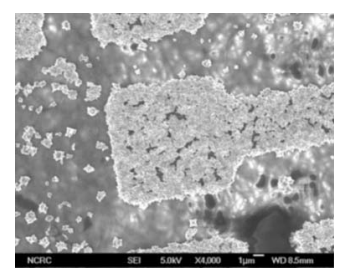

(b)

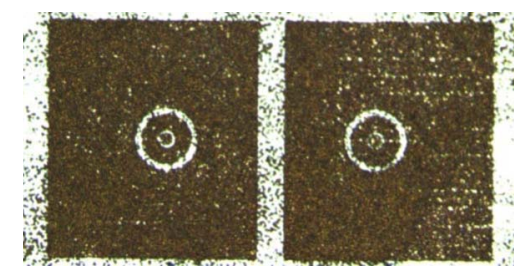

(c)

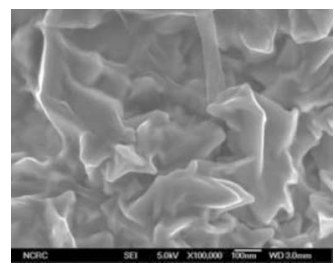

Figure 6. (Color online) The SEM and $\mathrm{OM}$ images of the electrode. Au was plated for $2 \mathrm{~h}$ at $50^{\circ} \mathrm{C}$ after SWCNT dip coating of the Pd-activated (condition A) (a) M1B- and (b) M2-type chips. (c) The SEM images of M1B electrodes. The Auplated chip (for $90 \mathrm{~min}$ at $50^{\circ} \mathrm{C}$ ) coated with SWCNT was Au-plated additionally for $15 \mathrm{~min}$ at $50^{\circ} \mathrm{C}$.
SWCNT has an electron-rich character, a high conductivity, and a defect coordination site, such as $-\mathrm{COOH},-\mathrm{OH}$, and $=\mathrm{CO} . \mathrm{Au}^{+}$ions have an electron affinity, which can interact with an electron-rich SWCNT.

1. Pd-activated M1B (or M2-type) sample: The Pd-activated sample (condition A) was dip coated with SWCNT before Au decoration. Then, Au ELP was done for $2 \mathrm{~h}$ at $50^{\circ} \mathrm{C}$. Figure $6 \mathrm{a}$ and $\mathrm{b}$ shows the FESEM and OM images of the electrode. Au was plated on the Al electrode as well as on the SWCNT net. Specifically, Au particles were decorated on SWCNT rather than the oxide surface in the TEOS layer. A SWCNT decoration study is underway to improve the $\mathrm{Au}$ particle size control and uniformity by this kind of $\mathrm{Au}$ ELP method.

2. Pd-activated and Au-plated M1B samples: A Au-plated M1B sample (Fig. 3e) was coated with SWCNTs, and again Au ELP was performed for $15 \mathrm{~min}$ at $50^{\circ} \mathrm{C}$. Figure $6 \mathrm{c}$ is the FESEM images of $\mathrm{Au}$ electrodes. Au clusters were formed on the SWCNT as well as the Au electrode surface. SWCNTs are under the Au cluster on the $\mathrm{Au}$ electrode as a result of surplus Au ELP. The resistivity of the SWCNT film was further reduced by more than twofold through a $\mathrm{Au}$ decoration process.

A sensor platform based on the metal decorated SWCNT.- For a sensor application, we decorate SWCNT with a Au particle using the upper Au ELP technique because gold nanoparticles deposited on solid supports exhibit various useful catalytic and electrocatalytic properties. ${ }^{9-12}$ Moreover, the surface of gold can be easily utilized as a substrate or a linker for a biomolecular sensor application by attaching the end of a thiol group of probe molecules.

Several electrical biosensor structures based on CNT have been reported, but our CMOS-compatible sensor platform integrated with SWCNT net and Au particles may be more useful because the Au particles on the SWCNT net as well as Au-plated electrode can be used as a substrate. It is believed that this synergy effect causes the remarkable molecular detection sensitivity ( $R$ increase) by modulating both the Au particles-SWCNT and Au electrode-SWCNT junctions. We have developed a CMOS-based DNA sensor with improved detection sensitivity by the SWCNT decoration with metal $(\mathrm{Au})$ particles $\left(\Delta R_{\mathrm{t}} / R_{\mathrm{p}}\right.$ : over $100 \%$ increase for $1 \mathrm{~V}$ applied across the electrodes; $p=25$ mer thiolated probe DNA, $\mathrm{t}$ is the complementary target DNA, and $R$ is the resistance). Furthermore, we have developed a gas sensor $\left(\mathrm{H}_{2}, \mathrm{CO}, \mathrm{NH}_{3}, \mathrm{H}_{2} \mathrm{~S}, \mathrm{C}_{2} \mathrm{H}_{5} \mathrm{OH}\right.$, and volatile organic compounds) using this platform by decoration of SWCNT with gas sensitive metals, such as $\mathrm{Sn}$ and $\mathrm{Pd}$. The sensor systems show the outstanding changes in conductivity $\left(R_{\text {air }} / R_{\text {gas }}\right)$ according to gas adsorption and desorption, whose data are subject to future publications.

\section{Conclusion}

To improve the contact resistance between the metal electrodes and the SWCNTs, we have developed the procedure of selective Au electroless deposition on a patterned $\mathrm{Al}$ electrode on CMOS-based chips, which is compatible with the conventional CMOS process without using any additional mask. The $\mathrm{Pd}$ activation of $\mathrm{Al}$ surface is accomplished by controlled removal of the $\mathrm{Al}$ oxide with phosphoric acid, which results in the formation of fine Pd particles on $\mathrm{Al}$. Subsequent Au ELP allowed selective deposition of the Au film on the Pd-activated Al surface. The SWCNT nets were integrated on the Au-plated CMOS-based chips using a dip-coating method, and the improved contact resistance and small resistance variation between the Au electrode and SWCNTs have been obtained. We also showed a SWCNT decoration with Au particles using an upper Au ELP method. This result is expected to be a suitable alternative to conventional Au ELP techniques as well as a sensor platform for fusion nanotechnology.

\section{Acknowledgment}

This work was supported by the Nano Systems Institute-National Core Research Center (NSI-NCRC) program of NRF and the TDPAF, Ministry for Agriculture, Forestry and Fisheries, Republic of Korea.

Seoul National University assisted in meeting the publication costs of this article.

\section{References}

1. B. L. Allen, P. D. Kichambare, and A. Star, Adv. Mater, 19, 1439 (2007)

2. W. Yang, P. Thordarson, J. J. Gooding, S. P. Ringer, and F. Braet, Nanotechnology, 18, 412001 (2007).

3. J. Kong, N. R. Franklin, C. Zhou, M. G. Chapline, S. Peng, K. Cho, and H. Dai, Science, 287, 622 (2000).

4. D. W. Kim, G. S. Choe, S. M. Seo, J. H. Cheon, H. Kim, J. W. Ko, I. Y. Chung, and Y. J. Park, Appl. Phys. Lett., 93, 243115 (2008).

5. S. J. Tans, A. R. M. Verschueren, and C. Dekker, Nature (London), 393, 49 (1998)

6. H. W. C. Postma, T. Teepen, Z. Yao, M. Grifoni, and C. Dekker, Science, 293, 76 (2001).

7. E. S. Snow, J. P. Novak, P. M. Campbell, and D. Park, Appl. Phys. Lett., 82, 2145 (2003).

8. H. Watanabe and H. Honma, J. Electrochem. Soc., 144, 471 (1997).

9. M. M. Maye, Y. Lou, and C. J. Zhong, Langmuir, 16, 7520 (2000).

10. S. R. Brankovic, J. X. Wang, and R. R. Adžić, Electrochem. Solid-State Lett., 4 , A217 (2001)

11. Y. Zhang, S. Asahina, S. Yoshihara, and T. Shirakashi, Electrochim. Acta, 48, 741 (2003).

12. K. Y. Chan, J. Ding, J. Ren, S. Cheng, and K. Y. Tsang, J. Mater. Chem., 14, 505 (2004).

13. D. A. Hutt, C. Liu, P. P. Conway, D. C. Whalley, and S. H. Mannan, IEEE Trans. Compon. Packag. Technol., 25, 87 (2002).

14. M. Liebau, E. Unger, G. S. Duesberg, A. P. Graham, R. Seidel, F. Kreupl, and W. Hoenlein, Appl. Phys. A: Mater. Sci. Process., 77, 731 (2003).

15. S. G. Robertson, I. M. Ritchie, and D. M. Druskovich, J. Appl. Electrochem., 25, 659 (1995).

16. T. Pearson and S. J. Wake, Trans. Inst. Met. Finish., 75, 93 (1997).

17. D. S. Lashmore, Plat. Surf. Finish., 65, 44 (1978).

18. E. Stoyanova and D. Stoychev, J. Appl. Electrochem., 27, 685 (1997).

19. S. W. Court, B. D. Baker, and F. C. Walsh, Trans. Inst. Met. Finish., 78, 157 (2000).

20. W. L. Goh and K. T. Tan, Thin Solid Films, 462-463, 275 (2004).

21. H. C. Koo, S. Y. Kim, S. K. Cho, and J. J. Kim, J. Electrochem. Soc., 155, D558 (2008).

22. J. C. Patterson, C. Ni Dheasuna, J. Barrett, T. R. Spalding, M. O'Reilly, X. Jiang, and G. M. Crean, Appl. Surf. Sci., 91, 124 (1995).

23. E. Y. Jang, T. J. Kang, H. W. Im, D. W. Kim, and Y. H. Kim, Small, 4, 2255 (2008).

24. Y. I. Song, G. Y. Kim, H. K. Choi, H. J. Jeong, K. K. Kim, C.-M. Yang, S. C. Lim, K. H. An, K. T. Jung, and Y. H. Lee, Chem. Vap. Deposition, 12, 375 (2006)

25. K. R. Williams, K. Gupta, and M. Wasilik, J. Microelectromech. Syst., 12, 761 (2003).

26. T. S. Olson, P. Atanassov, and D. A. Brevnov, J. Phys. Chem. B, 109, 1243 (2005).

27. S. T. Rao and R. Weil, J. Electrochem. Soc., 127, 1030 (1980).

28. Y. G. Li and A. Lasia, J. Appl. Electrochem., 27, 643 (1997).

29. M. J. O'Keefe, K. D. Leedy, J. T. Grant, M. Fang, H. Gu, and T. J. O'Keefe, J. Vac. Sci. Technol. B, 17, 2366 (1999).

30. See EPAPS supplementary material at: http://dx.doi.org/10.1149/1.3244208 E-JESOAN-157-001001 for additional information. 